\title{
Using HEP Expertise for Social \& Humanitarian Impact
}

\section{Daniel Dobos ${ }^{1}$}

GluoNNet

Avnue de Secheron 15, 1202 Geneva, Switzerland

E-mail: daniel@gluonnet.org

\section{Agnes Jakab}

Terre des Hommes

Avenue des Montchoisi 15, 1006 Lausanne, Switzerland

E-mail: agnes.jakabetdh.ch

\section{Ines Knaepper}

CERN

Esplanade des Particules 1, 1211 Geneva 23, Switzerland

E-mail: ines.knaepperecern.ch

\section{Elisabeth Pfund}

GluoNNet

Avnue de Secheron 15, 1202 Geneva, Switzerland

E-mail: elisabethegluonnet.org

\section{Karolos Potamianos}

$D E S Y$

Notkestraße 85, 22607 Hamburg, Germany

E-mail: karolos.potamianosecern.ch

\begin{abstract}
When humanitarian and social challenges from the United Nations, Red Cross and Non-Governmental Organisations meet HEP expertise impactful innovation becomes reality. THE Port association at CERN combines physicists and engineers working on HEP topics in their day job with researchers, refugees, entrepreneurs, artists, designers, humanitarian workers and other creative minds. During 60-hour-long curated hackathons they co-create new technology opportunities, identify new methods, materials and processes, that can be used in the humanitarian context and sometimes even feedback into HEP. Examples from the last 5 humanitarian hackathons at CERN, whose outcomes are now utilised by UN, ICRC and others as well as future initiatives for HEP society impact, are presented.
\end{abstract}

The 39th International Conference on High Energy Physics (ICHEP2018)

4-11 July, 2018

Seoul, Korea

\footnotetext{
${ }^{1}$ Speaker 


\section{Introduction}

Expanding our horizons and improving our knowledge in High-Energy Physics (HEP) is the main motivation for the fundamental research carried out at the European Organisation for Nuclear Research (CERN) and at other institutes.

The benefits from this research, however, need not be limited to the field of HEP, and can benefit other areas of society. Successfully using methodologies and processes developed in HEP research on challenges from other fields demonstrates the value of fundamental research to society. This is an important aspect, which strengthens the case for HEP funding, providing additional benefits to society, for example outreach, and answers the question "What's in it for the general public?" that donors and funding agencies tend to ask more and more, seeking to maximise the impact of their contribution.

Thus, interdisciplinary diversity opens up a new natural way of delivering decision makers arguments they need to support fundamental research.

\section{Outreach stories from different perspectives}

The excellent HEP outreach community impressively shows to a large audience, from the general public to policy and decision makers, what their work and passion is about. They proudly present how basic research is not only advancing the understanding of the world. However in most cases the outreach story is told from the HEP perspective. While visiting the world's leading HEP laboratories, visitors get explained what research is about and how it is being done. Often, the story goes: "A new tool or method was needed to better understand the world, so developments into this direction was started. While building and using those tools, it was discovered, that these learnings could be beneficial to society either." Well known examples are the World Wide Web, capacitive touch screens or solar collectors, just to name a few examples from CERN.

A complementary approach is to present the impact of fundamental science to other areas of society and demonstrate the benefits of research. This story helps reach donors and policy makers effectively.

THE Port association, based in Geneva, Switzerland, helps the HEP community to show immediate impact of basic research to society through their short co-creation events, in particular through their humanitarian hackathons. Driven by needs, problems and issues of humanitarian field workers are explored by multidisciplinary teams combining HEP scientists with experts from other domains, e.g. architects, artists, designers, engineers, entrepreneurs, students and other disciplines, to advance the outcome of those humanitarian projects in an innovative way.

Through interdisciplinary diversity and co-creation it can be demonstrated that HEP methods and technology helps to overcome humanitarian challenges. Hereby the value is shown directly in the domain of the decision makers. If, for example, a Secretary of Health and Human Service or a Minister of Housing and Urban Development understands the value of HEP for their field - even without the need to understand what HEP is or how it works - the goal is reached. It furthermore extends the spectrum of the audience to people, that are not interested in HEP or even in fundamental science in general. This only becomes possible by moving the comfort zone from the current HEP community approach towards the stakeholder's comfort 


\section{Using HEP Expertise for Social \& Humanitarian Impact}

zone and helps connecting the HEP tools and methods to challenges faced in other domains. Through co-creation diversity and inclusion turn from a moral obligation into a fundamental necessity.

\section{Methodology}

THE Port [1] organises curated humanitarian hackathons, that differ from classical hackathons by a thorough preparation of challenges, teams, mentors and a six-weeks preparation phase prior to the hackathon event.

Challenges are proposed by International Organisations, Non-Governmental Organisations or individuals and are shaped with them throughout a 6 months challenge definition and shaping process prior to the hackathon.

Hackathon participants are selected from a large pool of candidates, who submit their application which includes a Curriculum Vitae and answers to a few questions probing their background and motivation. All applications are reviewed and ranked by a seasoned interdisciplinary selection group. After an initial ranking, roughly double the amount of profiles as available places candidates are invited to answer three additional motivational questions using a web video interviewing tool.

Successful candidates are assembled by the selection group into interdisciplinary and balanced teams in gender, age and available professional disciplines, following the guiding principle, that each team member should be able to contribute to the success of the team. In order to balance between fresh ideas and experience, THE Port aims at achieving a similar age distribution as present at CERN.

Prior to the actual 60-hours hackathon event, a six-week-long preparation phase takes place, giving the newly assembled teams the possibility to learn more about the context of their challenge during some self-study time, using the advice of leading experts and mentors from all over the world about the field of their challenge. Because the teams include participants from all over the world, we rely heavily on video conferencing tools for this phase. Exposing the teams to field experts - not only during the virtual preparation phase but also during the event - gives valuable insights, directly answers many questions the team might have and ensures that the proposed opportunities are useful in the humanitarian context and environment. Including those leading experts from all over the world, e.g. ESA Astronauts, Nobel Prize Laureates, Humanitarian Key Actors or Ambassadors throughout the entire hackathon activity ensures, that the outcome of the challenges are taken forward by the right stakeholders and proposed solutions will actually be used by the relevant users.

The hackathon event is the moment, when the team meets in person for the first time in a truly inspiring place: CERN's IdeaSquare, a newly established fast prototyping facility. To allow the teams to focus on their challenge and start the first constructions of prototypes, the event agenda is kept light. Important steps are however the mid-term review, during which the teams present their status and plans, allowing immediate feedback from experts and other participants, and the final presentation, during which the teams showcase the outcome of the hackathon to a larger audience (local and over the Internet) at CERN's Globe of Science and Innovation.

A key factor is the broad interdisciplinary expertise and infrastructure available at CERN, allowing to spontaneously find technology experts on almost any given topic, such as, e.g., using the expertise of CERN's fire brigade or performing chemical analysis. 


\section{Using HEP Expertise for Social \& Humanitarian Impact}

Following THE Port's philosophy, the humanitarian hackathons foster collaboration, not competition. All challenges address pressing humanitarian needs; all challenges are considered equally important. Thus all teams get the opportunity to present their outcome during the final presentation to humanitarian and technology field experts. The final presentations are mostly followed-up by a presentation at a high-level event of relevant need-owners or other stakeholders, like during a side event at the $31^{\text {st }}$ Human Rights Council at the United Nations or at the opening at the Geneva Health Forum in 2016 and 2018. Since its foundation in 2014 THE Port has meanwhile curated more than 50 challenges in 16 events welcoming over 1000 participants.

\section{Outcomes}

From the over 50 challenges that THE Port has hosted in their hackathon events, some have been further developed after the 60-hours long innovation sprint. A few of them are introduced.

Taking for example the "Better Body Bag" [2] challenge using innovative materials and technologies into body bags has been developed further receiving funding from the Humanitarian Innovation Fund to bring the proposed solutions to the next level.

Solutions from the "Smart Dog" challenge improving the search effectiveness of demining dogs have been converted to a useable product and have been started to be produced by a Swiss company currently testing the product in the field with humanitarian key actors in the demining of land mines world.

To better protect children suffering from UV Hypersensitivity, the challenge team has developed a detector for sun rays allowing the children to remove their astronaut-like helmets if no sun light in a room has been detected.

\section{Visual Analytics for Human Aid}

Another concrete outcome of interdisciplinary co-creation is the gluoNNet association [3]. Human aid (humanitarian, development and human rights) organisations expressed the need for better answers on how to break silo thinking in international collaborations. GluoNNet aims to analyse collaborations using big data and visual analytics technologies. It is based on Collaboration Spotting [4] algorithms developed by CERN in the context of the Large Hadron Collider (LHC).

Fig. 1 is an example of an ecosystem knowledge graph retrieved from worldwide scientific publications and patents showing communities or collaboration between

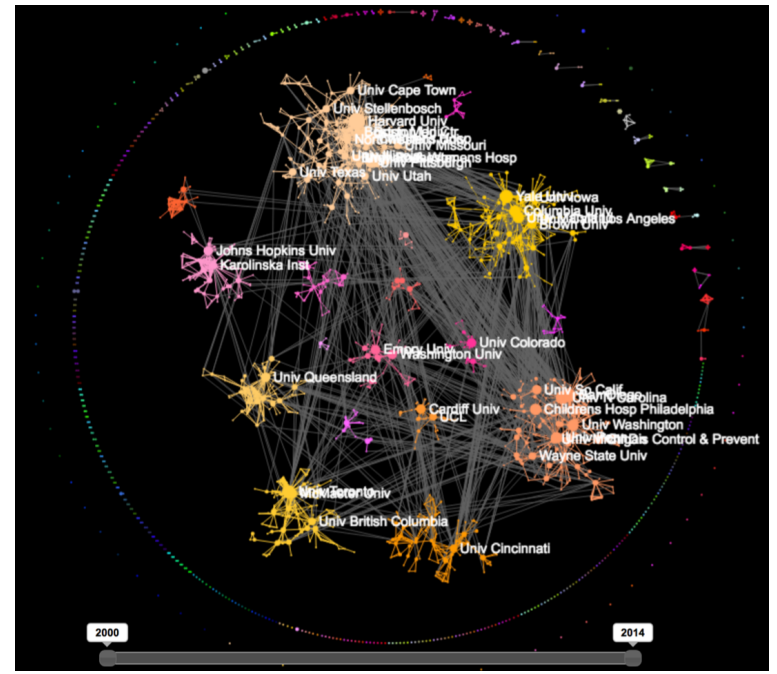

Figure 1 - Mobile Health ecosystem knowledge graph created with CERN's Collaboration Spotting showing communities of collaboration between research institutes and companies. 


\section{Using HEP Expertise for Social \& Humanitarian Impact}

research institutes and companies on the human aid topic of mobile health. GluoNNet's goal is that such types of literature reviews save time, provide confidence in a knowledge overview and uncover unexpected insights for smart decisions.

These tools empower to overcome limits of personal networks \& information overflow, so decision makers can save critical time, have a life-changing impact and be the heroes that our world needs.

\section{Conclusions}

Diverse, inclusive and interdisciplinary co-creation helps to demonstrate that tools and methods developed for HEP become beneficial to society, for example in the humanitarian context.

\section{References}

[1] http://www.theport.ch

[2] "Benefits of diverse and interdisciplinary co-creation for HEP - a showcase", D. Dobos et al, PoS(ICHEP2016)312, July 2016

[3] http://www.gluonnet.org

[4] “Collaboration Spotting: A Visual Analytics Platform to Assist Knowledge Discovery", A. Agocs, D. Dardanis, R. Forster, J.-M. Le Goff, X. Ouvrard and A. Rattinger, in ERCIM News 111, Research and Innovation, September 2017 